\title{
Estudio de la energía superficial y hemocompatibilidad del Ti y TiN sintetizados por Magnetron Sputtering para aplicaciones médicas
}

\author{
Study of surface energy and hemocompatibility of Ti and TiN synthesized by \\ Magnetron Sputtering for medical application
}

\author{
Diana Alexandra Gómez ${ }^{1}$, Alexander Ruden², Juan Manuel González ${ }^{3}$, Federico Sequeda ${ }^{4}$ \\ ${ }^{1}$ Ingeniera de Materiales, Grupo de Investigación: Laboratorio de Recubrimientos Duros y Aplicaciones \\ Industriales (RDAI), Escuela de Ingeniería de Materiales, Universidad del Valle, Valle del Cauca, Colombia. \\ dalexandragomezegmail.com \\ ${ }^{2}$ Ph.D. Docente, Grupo de Investigación: Laboratorio de Recubrimientos Duros y Aplicaciones Industriales \\ (RDAI), Facultad de Ciencias Básicas, Universidad Tecnológica de Pereira, Risaralda, Colombia. \\ arudenmeutp.edu.co \\ ${ }^{3}$ Ph.D. Grupo de Investigación en Desarrollo de Materiales y Productos GIDEMP, Centro ASTIN, SENA \\ regional Valle del Cauca, Laboratorio de Recubrimientos Duros y Aplicaciones Industriales (RDAI), \\ Universidad del Valle, Valle del Cauca, Colombia. \\ juanmonzalezcegmail.com \\ ${ }^{4}$ Ph.D. Docente, Grupo de Investigación: Laboratorio de Recubrimientos Duros y Aplicaciones \\ Industriales (RDAI), Escuela de Ingeniería de Materiales, Universidad del Valle, Valle del Cauca, Colombia. \\ fsequeda@correounivalle.edu.co
}

\begin{abstract}
Resumen-Se simularon computacionalmente y sintetizaron los materiales Ti hexagonal y TiN fase cubica, para mostrar su posible aplicaciones como biomaterial. Las simulaciones computacionales realizadas utilizando Teoría de Funcionales de Densidad (DFT) muestran la tendencia hidrofóbica de estos materiales lo que los hace candidatos para una aplicación biomédica, por la facilidad de interacción con el tejido humano. Por medio de la técnica Magnetron Sputtering DC, se sintetizaron los recubrimientos de $\mathrm{Ti}$ y $\mathrm{TiN}$ sobre sustratos biocompatibles de acero inoxidable AISI 316L, analizando la hemocompatibilidad; se demostró que el recubrimiento de $\mathbf{T i}$ posee mayor energía interfacial lo cual promueve alta probabilidad de formación de trombos respecto al sustrato biocompatible 316L y el recubrimiento de TiN.
\end{abstract}

Palabras clave - biomateriales, hemocompatibilidad, simulación computacional.

Abstract - The materials hexagonal-Ti and cubic phase-TiN were computationally simulated and synthesized, in order to determinate their potential applications as biomaterials. Computer simulations preformed using Density Functional Theory (DFT) showed a hydrophobic tendency, which makes them candidates for biomedical applications. Using magnetron sputtering DC technique, the $\mathrm{Ti}$ and $\mathrm{TiN}$ coatings were synthesized on biocompatible AISI 316L stainless Steel substrates analyzing hemocompatibility; It was proven that the Ti coating has a higher interfacial energy which may lead to increased probability of thrombus formation with respect to the biocompatible substrate 316L and TiN.

Key Word - Biomaterials, Hemocompatibility, computational method.

\section{INTRODUCCIÓN}

Los Biomateriales metálicos, son una gama de materiales formado por uno o más metales en aleación, algunos con la capacidad de alojar materiales de carácter no metálicos en su estructura, como boro, oxígeno y nitrógeno; ejemplo de estos últimos, dióxido de titanio $(\mathrm{ZrO} 2)$ y nitruro de circonio $(\mathrm{ZrN})$ $[1,2]$. Para el caso de materiales metálicos en general, poseen organización específica con enlace metálico. En cuanto a biomateriales metálicos, normalmente son aquellos fabricados en acero inoxidable, aunque en la actualidad se trata de aleaciones, de tal forma que mejore su estatus de biocompatible [3]; su uso es limitado ya que con el contacto 
con medios agresivos como el tejido humano y sangre produce procesos de ionización, que se dan por disolución, galvanismos o corrosión. Hoy día los más usados son aleaciones ferrosas con alta resistencia a la corrosión por la incorporación de cromo al menos de un $12 \%$ y algunas no ferrosas como las aleaciones de $\mathrm{Mg}$, Ti y $\mathrm{Ni}$, donde las de $\mathrm{Ti}$ y Ni tiene alta aplicación en medicina. Las aleaciones de Ti, aunque costosas, poseen propiedades de alta resistencia mecánica, además de ser muy ligeras. Los materiales cerámicos, adquieren sus propiedades específicas por el tipo de enlace químico que poseen (iónico y covalente); dentro del mundo de los materiales cerámicos, hacen parte de la gama de los más complejos en su estudio, ya que pueden poseer propiedades mecánicas, ópticas, semiconductoras o superconductoras, las cuales se debe a sus elementos aleantes de carácter metálico como no metálico. En cuanto a sus propiedades básicas en aplicaciones biocompatibles, los cerámicos son duros, frágiles con baja tenacidad y ductilidad, presentan altos puntos de fusión y bajas conductividades eléctrica y térmica, en la mayoría de los casos se comportan con relativa estabilidad en ambientes agresivos, debido al equilibrio de sus fuertes enlaces. Se usan biomateriales cerámicos para la fabricación de prótesis e implantes quirúrgicos o para cumplir una actividad fisiológica en el cuerpo humano ya que poseen alta biocompatibilidad; Algunos de estos actúan como osteointegradoras por su similar composición a la del sistema óseo [4], aunque tiene limitaciones en su aplicación por su alta rigidez, lo que conlleva a ser quebradizos cuando actúan altas cargas en la aplicación. En el análisis de materiales de aplicación biomédica, se encuentran aquellos que usan el titanio, sea en estado puro, o en aleaciones, para diversos tipos de implantes, en especial dentarios, cambios de partes de cadera, o el uso de aleaciones inteligentes [5], con aplicaciones desde ortodoncia hasta ortopedia, usando su propiedad de memoria de forma. El Ti tiene estrecha afinidad con el nitrógeno, creando compuestos intersticiales, cerámicos de denominación avanzada, que poseen mejores propiedades mecánicas como dureza [6]; el compuesto de este tipo, nitruro de titanio (TiN), tiene superiores propiedades, mecánicas, químicas y tribológicas, por tanto es usado como tratamiento superficial de distintos aceros usando técnicas asistidas por plasma [7] que posibilitan un aumento en la inercia química, dureza y disminuyen valores de daño por contacto tribológico (desgaste) [8]. Las primeras interacciones entre superficie y tejido biológico, están relacionadas con la acción del agua y proteínas. Cuando el biomaterial se pone en contacto con los fluidos y tejidos, la molécula de agua en su estado líquido se pone en contacto con la superficie, este proceso tiene tiempo de acción del orden de los nanosegundos, formando monocapas o bicapas de agua con estructura diferente a la de su estado líquido. Si la superficie es hidrofílica, el agua es disociada formando una superficie terminada en el grupo funcional hidroxilo $(-\mathrm{OH})$ fuertemente adherida a la superficie del material, si por lo contrario es hidrofóbica la adherencia es poca sobre la superficie. El contenido básico del tejido y fluidos corporales es el cloruro de sodio $(\mathrm{NaCl})$, en su forma iónica: $\mathrm{Na}+$ y $\mathrm{Cl}$-, donde estos iones hidratados son atraídos por acción electrostática sobre la superficie con capa hidroxílica, formando lo que se conoce como doble capa, para después dar paso a las proteínas, las cuales interaccionarán con la superficie del biomaterial, dando paso a los procesos celulares. El presente trabajo muestra el estudio de la energía superficial de los materiales Ti y TiN desde dos puntos de vista, (a) teórico (simulación de la celda cristalina) y (b) experimental, usando el método de Owens - Wendt - Kaeble, midiendo el ángulo de mojado sobre los recubrimientos sintetizados por la técnica Magnetron Sputtering sobre acero inoxidable AISI 316L.

\section{METODOLOGIA}

Método computacional Usando métodos computacionales, con algoritmos electrodinámicos y mecano cuánticos (Teoría de Funcionales de Densidad-DFT), se simularon con el software GAUSSIAN 3 [9], las estructuras moleculares de los recubrimientos a discutir, en nuestro caso capas delgadas de Ti fase hexagonal y TiN fase cúbica. El sistema GAUSSIAN 3 (licencia número PC21297755V-5229N perteneciente al laboratorio de Recubrimientos Duros y Aplicaciones Industriales (RDAI) de la Universidad del Valle); se utilizó un porcentaje de memoria operativa de $6 \mathrm{MW}$, soportado por una estación de trabajo HP dc7900 Smalll Form Factor, calculando así en forma aproximada los valores propios de la energía y las autofunciones del espectro discreto y el método Hartree - Fok de campo autoconsistente [10] calculando así de forma ascendente o descendente las energías de los estados fundamentales de átomos con varios electrones. El método variacional, supone un sistema no degenerado con energía $E_{1}$, que es la más baja de un sistema, con autofunción $\varphi_{1}$, obedeciendo al teorema de Ritz [11] que afirma (ec. 1): Si H es operador hermítico, con espectro discreto y acotado inferiormente (ec. 2) y dado $\mathrm{E}$ un funcional:

$$
E: \Psi \in F \rightarrow E \Psi=\frac{\langle\Psi|H| \Psi\rangle}{\langle\Psi \mid \Psi\rangle}
$$

Entonces:

$$
E \Psi \geq E_{1} \forall \Psi \in F \text { por tanto } \Psi=\varphi_{1}
$$

Concluyendo, la minimización de un funcional, conduce a la búsqueda de los estados correspondientes de la energía H. Las aproximaciones de Hartree Fok, conecta la simetría de intercambio de las funciones de onda del sistema, donde hay interacción de muchos fermiones que interactúan de forma cualquiera. Cuando existe un conjunto de $\mathrm{n}$ fermiones, la descripción cuántica del Hamiltoniano (ec. 3) está dada por la expresión:

$\widehat{H}=\sum_{\alpha \alpha 1} h_{\alpha}^{1 \alpha 1} b_{\alpha 1}^{+} b_{\alpha}+\frac{1}{2} \sum_{\alpha \beta \alpha 1 \beta 1} v_{\alpha \beta}^{2 \alpha 1 \beta 1} b_{\alpha 1}^{+} b_{\beta 1}^{+} b_{\alpha} b_{\beta}$

Dónde: $b_{\alpha}$ y $b_{\alpha}^{+}$son los operadores aniquilación y creación de un sistema ortonormal completo, encontrando las nuevas 
autofunciones del sistema, dado por $\Psi_{o}$, para la cual el valor de la energía sea mínimo (estado fundamental); para el resto de estados $\Psi_{n}$ (ec. 4) se usa los operadores convencionales de aniquilación y creación ( $\widehat{a})$, obteniendo [12]:

$$
\Psi_{n}=\hat{a}_{n}^{+} \hat{a}_{n-1}^{+} \hat{a}_{n-2}^{+} \ldots \hat{a}_{2}^{+} \hat{a}_{1}^{+} \Psi_{0} \quad(\text { ec. } 4)
$$

En la tabla 1 se muestra la variables: tamaño de enlace correspondientes a las simulaciones de las unidades moleculares de cada sistema; valores tomados de los elementos en estado puro (tabla periódica). El cálculo computacional tiene criterio de convergencia de funciones de estado, con valor de $3 * 10-12$ unidades atómicas de energía (UAE).

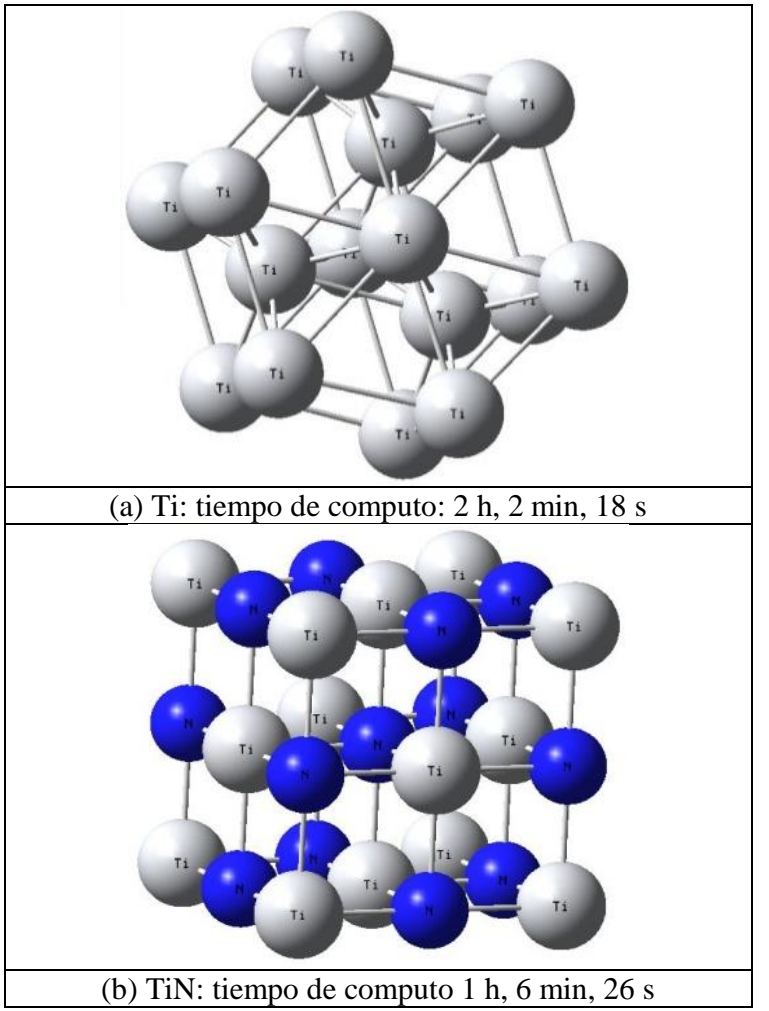

Figura 1. Estructura cristalina de las moléculas simuladas. (a) Ti; (b) $\mathrm{TiN}$

Tabla 1. Tamaño de enlace para las estructuras simuladas

\begin{tabular}{|c|c|}
\hline Material & Tamaño de enlace $(\AA)$ \\
\hline $\mathrm{Ti}$ & 2.94 \\
\hline $\mathrm{TiN}$ & 2,39 \\
\hline
\end{tabular}

En la figura 1a y 1b se muestra el arreglo cristalográfico a simular para la fase hexagonal del Ti y la fase cubica del TiN respectivamente, además de los tiempos de cómputo de la simulación.

Para la síntesis de recubrimientos en capa delgada de los diferentes materiales ( $\mathrm{Ti}, \mathrm{TiN})$, se utilizó un sistema Magnetron Sputtering DC: sistema multifuente Magnetrón
Sputtering PVD (AJA INTERNATIONAL ATC1500). Mencionados recubrimientos se depositaron sobre acero inoxidable austenítico AISI 316L; se utilizó barra de $1.25 \mathrm{~cm}$ de diámetro, fraccionada en cilindros de $4 \mathrm{~mm}$ de espesor, cuya superficie fue pulida, utilizando papel abrasivo de carburo de silicio con granulometría entre 700 - 2500 para generar terminado superficial especular; antes de ser inmersos en el reactor evaporador PVD - Sputtering, fueron sometidas a lavado ultrasónico por 15 minutos en fluido de acetona, eliminado agentes extraños como grasas y polvos, contaminación debida a manipulación. En la tabla 2, se muestran las condiciones de síntesis de estos recubrimientos. $\mathrm{El}$ espesor de estos recubrimientos fue constante de $570 \mathrm{~nm}$, con tiempos de síntesis de 60 min y $120 \mathrm{~min}$ para el Ti y TiN respectivamente.

Tabla 2 Condiciones de síntesis de los materiales en capa delgada de Ti, TiN.

\begin{tabular}{|c|c|c|c|c|}
\hline Material & $\begin{array}{c}\text { Blanco } \\
\text { precursor }\end{array}$ & $\begin{array}{c}\text { Presión } \\
\text { (mtorr) }\end{array}$ & $\begin{array}{c}\text { Flujo Ar } \\
(\mathbf{s c c m})\end{array}$ & $\begin{array}{c}\text { Flujo } \\
\mathbf{N}_{\mathbf{2}} \\
(\mathbf{S c c m})\end{array}$ \\
\hline $\mathrm{Ti}$ & $\begin{array}{c}\mathrm{Ti} \\
(99.99 \%)\end{array}$ & 3 & 10 & - \\
\hline $\mathrm{TiN}$ & $\begin{array}{c}\mathrm{Ti} \\
(99.99 \%)\end{array}$ & 3 & 10 & 0.15 \\
\hline
\end{tabular}

La capa delgada metálica de Ti depositada sobre acero AISI 316L usando Sputtering no reactivo, posee estequiometría equivalente al del material blanco precursor, el cual es hexagonal, certificado por la industria PLASMATERIALS S.A.; caso contrario ocurre para el TiN, donde se usó una mezcla de gas reactivo nitrógeno con argón y se caracterizó usando Difracción de Rayos X (XRD), usando un difractómetro Rigaku Última III, con radiación $\mathrm{CuK} \alpha$ $(\lambda=0,1540 \mathrm{~nm})$, operado a $40 \mathrm{kV}$ y $44 \mathrm{~mA}$, con geometría $\theta-$ $2 \theta$, en ángulos desde 30 hasta $80^{\circ}$.

Análisis energético (tensión superficial) La tensión superficial de los materiales se obtuvo por el método del ángulo de mojado, considerando la norma ASTM D7490 que se basa en el modelo matemático de Owens -Wendt - Kaeble (ec. 5), para lo cual se requiere dos líquidos con tensión superficial conocida al igual que sus contribuciones polar y dispersiva, en este caso agua destilada y glicerol; donde $\theta$ es el ángulo de contacto del líquido con la superficie del sólido, $\gamma_{l}$ la tensión superficial del líquido (dinas $/ \mathrm{cm}^{2}$ ) y $\gamma^{d}, \gamma^{p}$ la componente de dispersión y polar de los líquidos y sólidos.

$$
\frac{\gamma_{1}(1+\cos \theta)}{2}=\left[\left(\gamma_{l}^{s} \gamma_{s}^{d}\right)^{1 / 2}+\left(\gamma_{l}^{p} \gamma_{s}^{p}\right)^{1 / 2}\right]
$$

Para llevar a cabo el experimento se deposita una gota del líquido sobre la superficie del material y por medio de una cámara de alta resolución se toma una imagen digital del sistema, posterior a ello se realiza el cálculo del ángulo de mojado por un software de distribución libre de medida Meazure [13]. La figura 2 muestra el procedimiento. 


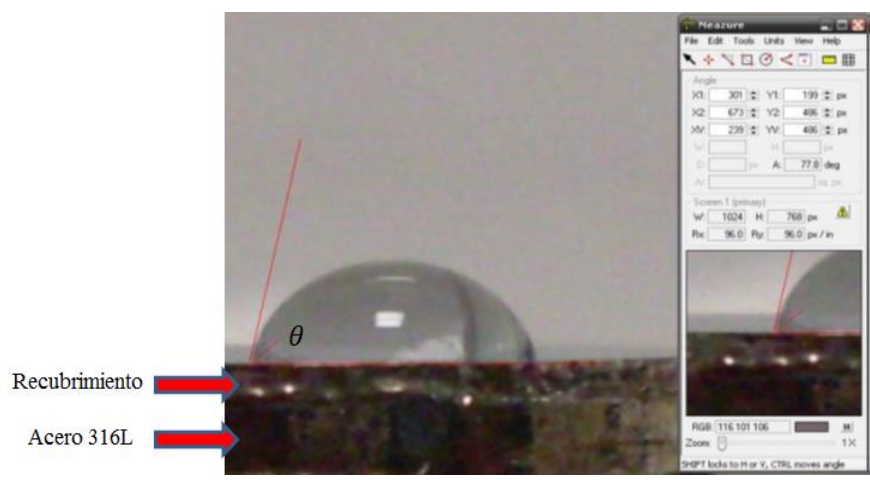

Figura 2. Procedimiento de medida del ángulo de mojado

A partir del sistema de ecuaciones formado debido a cada uno de los fluidos se calcula la componente dispersiva y polar del sólido; la tensión superficial del sólido se calcula como la suma de ambas componentes.

Hemocompatibilidad A partir de la prueba de adherencia y activación plaquetaria in vitro, se mostró la cantidad, morfología, agregación y pseudópodos de las plaquetas adheridas. En la figura 3, se puede apreciar el orden consecutivo de la obtención del plasma rico en plaquetas (PRP). La sangre se obtuvo de un voluntario adulto sano y libre de la aspirina u otros medicamentos que podrían sesgar los resultados (datos donante: Peso $=51 \mathrm{Kg}$, edad: 20 años, talla: $1.61 \mathrm{~m}$ ). Posterior a ello, por medio de centrifugación, se separan los glóbulos rojos y plaquetas y se obtiene el PRP.

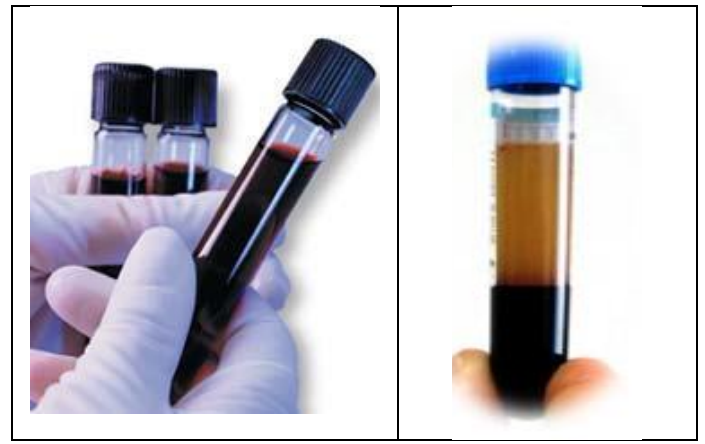

Figura 3. Sangre y plasma rico en plaquetas

Las muestras se sumergen en el PRP y se incuban a $37^{\circ} \mathrm{C}$ durante $48 \mathrm{~h}$; posterior a ello se procede a lavar las muestras con una solución de $\mathrm{NaCl}$ al $0,9 \%$ v/v para eliminar las plaquetas débilmente adheridas; se realizó Microscopia Electrónica de Barrido (SEM) en un equipo JEOL JSM-6480 $\mathrm{LV}$, con el fin de examinar la morfología de las plaquetas adheridas (X 1000 y X 2500).

\section{RESULTADOS Y ANALISIS}

\section{Análisis computacional}

A continuación se muestran las simulaciones de las estructuras moleculares de los recubrimientos de Ti y TiN en sus fases hexagonal y cubica respectivamente. En la figura 4 (a) y (b) se muestra la distribución de carga electrostática para para las estructuras cristalinas simuladas a través del software Gaussian 3. Dichas distribuciones se muestra para cada carga de los arreglos cristalográficos (cargas o electronegatividad de Mulliken) [14], estimando la estabilidad y neutralidad de estructura, confirmado la afinidad electrónica y potencial de ionización de los electrones de valencia en cada átomo, expresado bajo la suma promedio de carga con valor cero, hecho que se puede apreciar en la distribución de barras (figura 5); en cada uno de los casos se muestra la característica neutral de las estructuras, lo que indica la compatibilidad electroquímica entre los elementos que forman las celdas cristalinas. $(1$ Hartree $=2625.5 \mathrm{KJ} / \mathrm{mol}=27.212$ eV). En la figura 6 se muestra la densidad de carga electrónica para las estructuras del Ti hexagonal y TiN cúbico. Usando cálculos computacionales, se determinó la densidad de carga electrónica determinada por el arreglo de átomos en las estructuras, solucionando la ley de divergencia de campo eléctrico, donde se generó información sobre la distribución y signo de la carga eléctrica en un sistema de partículas a partir del cálculo de flujo de campo, donde se visualiza: Rojo $\rightarrow$ sección del sistema cargado negativamente y Azul $\rightarrow$ sección del sistema electropositivo. Para las simulaciones en general, se observa el carácter eléctricamente neutro. Este resultado es una condición electrostática optima, para la cual las estructuras presentan su menor energía de formación, por tanto mayor estabilidad. Se observan superficies con secciones electronegativas (color rojo sobre la superficie) lo que demuestra que las superficies tienen la tendencia a ser hidrofóbicas, lo que los hace buenos candidatos para una aplicación biomédica cuando el material este en contacto con el tejido humano, de tal forma que probablemente exista disociación del agua formando grupos $-\mathrm{OH}$ para posteriormente interactuar con proteínas [15]. La acción hidrofílica o hidrofóbica de una superficie en contacto con tejidos o fluidos corporales humanos tiene un papel importante en la aplicación del biomaterial, ya que un biomaterial hidrofóbico, aplicado por ejemplo en un stent coronario, evita la obstrucción de fluidos por compensación de carga electrostática, donde existe absorción de forma selectiva por acción de una baja fuerza iónica sobre el biomaterial hidrofílico.

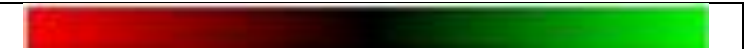




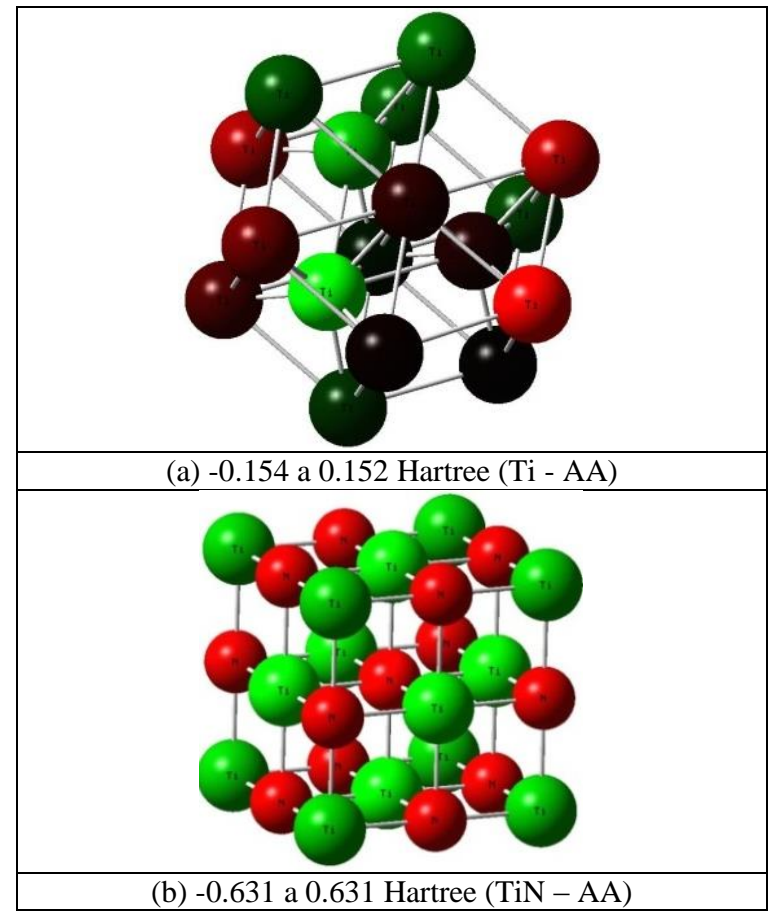

Figura 4. Simulación de la distribución de carga electrostática par las moléculas de Ti, TiN

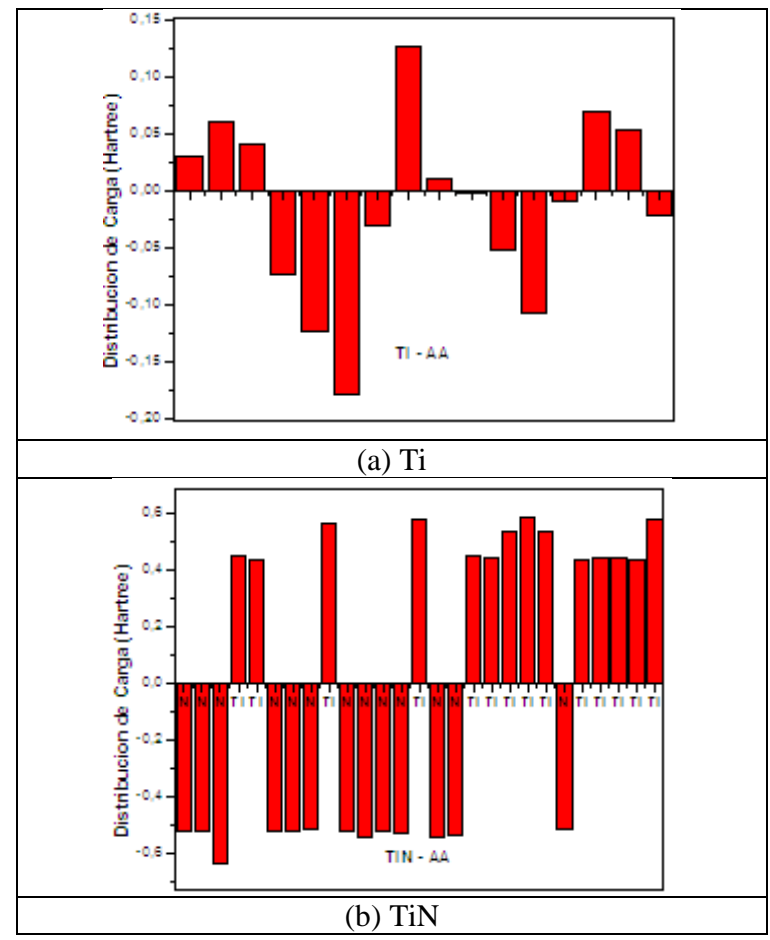

Figura 5. Diagrama de barras para la distribución de carga: estructuras moleculares de (a) Ti, (b) TiN.

Por tanto, desde el punto de vista teórico, los tratamientos en capa delgada utilizando los materiales $\mathrm{Ti}$ y $\mathrm{TiN}$ son de carácter hidrófobo y son aptos para aplicaciones en regeneración de hueso por medio de osteoblastos [16].

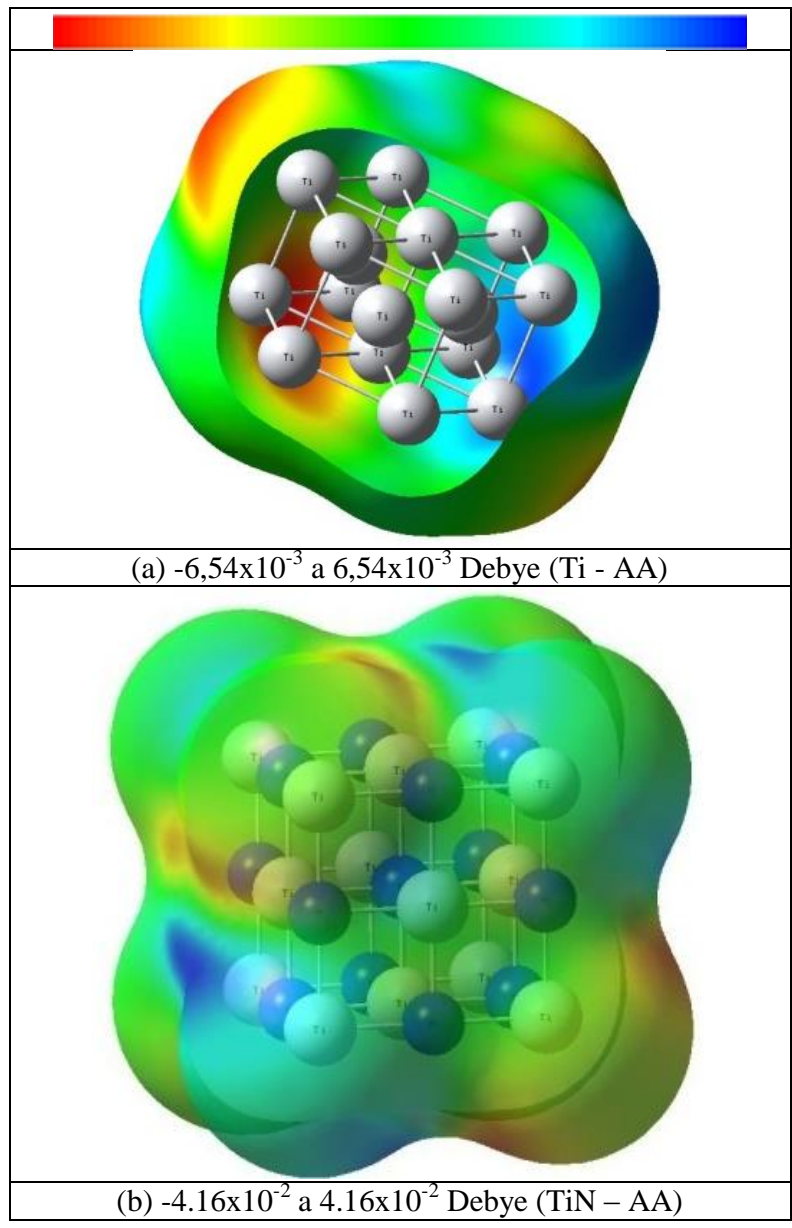

Figura 6. Simulación de la distribución de la densidad de carga para las moléculas de (a) Ti y (b) TiN

\section{Análisis estructural}

Las capas delgadas metálicas fueron depositadas sobre acero AISI 316L usando Sputtering no reactivo en atmosfera de argón, por tanto la estequiometria del recubrimiento es equivalente al del material blanco precursor, el cual es hexagonal, certificado por la industria PLASMATERIALS S.A., caso contrario ocurre para el nitruro de titanio (TiN), depositado utilizando una mezcla de nitrógeno reactivo con argón. Debido a esto la superficie recubierta con TiN se caracterizó usando XRD, obteniendo una estructura cúbica centrada en las caras. La figura 7 muestra el patrón de difracción del TiN, caracterizado por los picos correspondientes a los planos cristalográficos (111), (200), (220) y (311) en ángulos $2 \theta$ de: $34.2^{\circ}, 39.8^{\circ}, 59.1^{\circ}$ y $86.1^{\circ}$ respectivamente; dicha estructura ha sido estudiada por diferentes autores y es considerada como una estructura densa, con presencia de esfuerzos intrínsecos compresivos, alta densidad de dislocaciones y por tanto buen comportamiento mecánico [17]. 


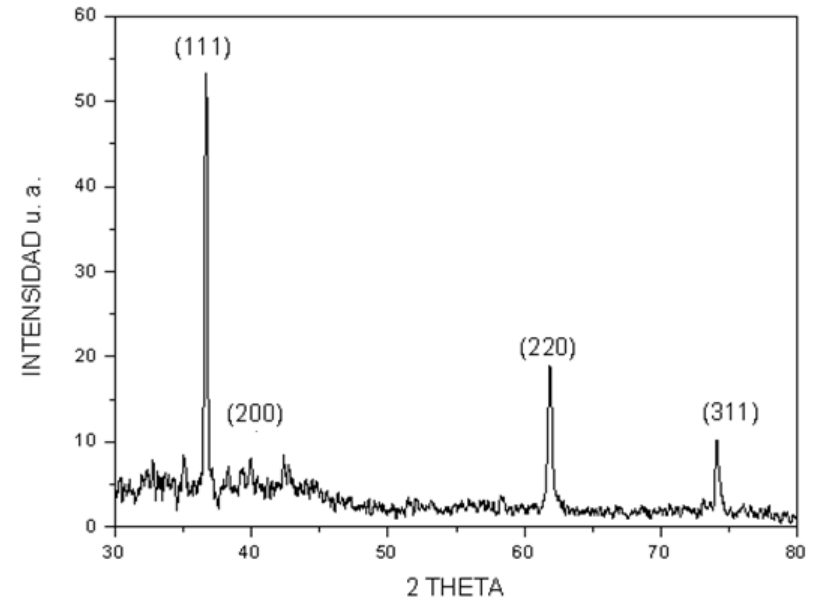

Figura 7. Patrón XRD para el TiN

\begin{tabular}{|c|c|c|c|c|}
\hline Fluido & $\begin{array}{c}\text { Agua } \\
\text { destilada }\end{array}$ & $\begin{array}{c}\text { Coef de } \\
\text { variación } \\
(\boldsymbol{\%})\end{array}$ & Glicerol & $\begin{array}{c}\text { Coef de } \\
\text { variación } \\
(\boldsymbol{\%})\end{array}$ \\
\hline Material & \multicolumn{4}{|l}{} \\
\hline $\mathbf{3 1 6 L}$ & $72.7^{\circ}$ & 1 & $80.9^{\circ}$ & 2 \\
\hline $\mathbf{T i}$ & $74.4^{\circ}$ & 1 & $73.7^{\circ}$ & 2 \\
\hline $\mathbf{T i N}$ & $65.6^{\circ}$ & 3 & $72.6^{\circ}$ & 2 \\
\hline
\end{tabular}

Tabla 5. Componentes polar y dispersiva de los materiales $316 \mathrm{~L}$, Ti y $\mathrm{TiN}$

\begin{tabular}{|c|c|c|c|}
\hline Material & $\begin{array}{c}\text { Componente } \\
\text { dispersiva } \\
\left(\mathbf{n J} / \mathbf{c m}^{\mathbf{2}}\right)\end{array}$ & $\begin{array}{c}\text { Componente } \\
\text { polar } \\
\left(\mathbf{n J} / \mathbf{c m}^{\mathbf{2}}\right)\end{array}$ & $\begin{array}{c}\text { Tensión } \\
\text { superficial } \\
\left(\mathbf{n J} / \mathbf{c m}^{\mathbf{2}}\right)\end{array}$ \\
\hline $\mathbf{3 1 6 L}$ & $2.50 \pm 0.03$ & $23.71 \pm 0.01$ & $26.21 \pm 0.02$ \\
\hline $\mathbf{T i}$ & $0.02 \pm 0.01$ & $41.45 \pm 0.01$ & $41.45 \pm 0.01$ \\
\hline TiN & $1.19 \pm 0.01$ & $34.59 \pm 0.02$ & $34.59 \pm 0.01$ \\
\hline Sangre & 11.2 & 36.3 & 47.5 \\
\hline
\end{tabular}

\section{Análisis tensión superficial y hemocompatibilidad}

Se analizaron las tensiones superficiales de los recubrimientos de Ti y TiN, junto con el sustrato de acero AISI 316L, usando dos fluidos, agua destilada y glicerol. Para el cálculo de tensiones superficiales (análisis energético) se determinaron las componentes polar y dispersiva midiendo los ángulos de mojado, cuyos valores de tensión superficial y componentes son conocidas, estos valores son registrados en la tabla 3 [18].

Tabla 3. Parámetros de la tensión superficial de los fluidos de prueba

\begin{tabular}{|c|c|c|c|}
\hline fluido & $\begin{array}{c}\text { componente } \\
\text { dispersiva } \\
\left(\mathbf{n J} / \mathbf{c m}^{\mathbf{2}}\right)\end{array}$ & $\begin{array}{c}\text { Componente } \\
\text { polar } \\
\left(\mathbf{n J} / \mathbf{c m}^{\mathbf{2}}\right)\end{array}$ & $\begin{array}{c}\text { Tensión } \\
\text { superficial } \\
\left(\mathbf{n} \mathbf{J} / \mathbf{c m}^{\mathbf{2}}\right)\end{array}$ \\
\hline $\begin{array}{c}\text { Agua } \\
\text { destilada }\end{array}$ & 21.8 & 51.0 & 72.8 \\
\hline Glicerol & 37.0 & 26.4 & 63.4 \\
\hline
\end{tabular}

La medida de ángulos de mojado (contacto) para los recubrimientos y sustrato de acero AISI $316 \mathrm{~L}$, se muestran en la tabla 4 , junto con la desviación porcentual de la medida angular (5 repeticiones).

El tratamiento de los datos se realizó en base al modelo de Owens - Wendt - Kaeble [19], de donde se despeja las componentes polar y dispersiva de los materiales, posterior a ello, la tensión superficial del material. En la tabla 5 se muestran los valores resultantes para cada capa delgada y sustrato, además la de la sangre, la cual se encuentra en la literatura [20].

A partir de la tensión superficial y sus respectivas componentes, se calculó la tensión interfacial solido - sangre, valores que se muestran en la tabla 6 .

Tabla 4. Parámetros de la tensión superficial de los fluidos de prueba

Tabla 6. Tensión interfacial Sangre - solidos

\begin{tabular}{|c|c|c|c|}
\hline $\begin{array}{c}\text { Sustancia } \\
\text { biológica }\end{array}$ & $\mathbf{3 1 6 L}$ & Ti & TiN \\
\hline Sangre & $7.20 \mathrm{~nJ} / \mathrm{cm}^{2}$ & $10.51 \mathrm{~nJ} / \mathrm{cm}^{2}$ & $5.25 \mathrm{~nJ} / \mathrm{cm}^{2}$ \\
\hline
\end{tabular}

En la tabla 6 se observa que el recubrimiento de Ti tiene la mayor energía interfacial con la sangre comparado con el sustrato de acero AISI 316L y el recubrimiento de TiN donde; estos resultados permiten inferir que la interacción del Ti con la sangre puede desencadenar con mayor probabilidad la formación de trombos con respecto al sustrato y el TiN, ya que la hemocompatibilidad es una propiedad que debe ser tenida en cuenta en implantes que requieren estar en contacto con la sangre, con el fin de que no se genere agregación de plaquetas en la superficie y se desencadene la formación de trombos, lo cual implica que el paciente sea tratado con antiagregantes para que no sufra algún evento cardiaco adverso [21, 22]. Mediante microscopía electrónica de barrido (SEM) se pudo observar la interacción de las superficies con las plaquetas de la sangre en los distintos materiales y así hacer un análisis cualitativo y cuantitativo de la interacción de los recubrimientos con el medio sanguíneo, pues es posible realizar el conteo de dicha células sobre las superficies expuestas. En las figuras 8 (a), (b) y (c), se muestran las micrografías de las plaquetas sobre la superficie del sustrato de acero inoxidable AISI 316L y los respectivos recubrimientos $\mathrm{Ti}$ y $\mathrm{TiN}$, en ellas se puede observar que las células se relacionan con las superficies, pues en todos los casos existió adhesión y agregación de las plaquetas, indiferentemente del tratamiento utilizado.

En el proceso de la activación de plaquetas se generan tres grandes cambios: adhesión de la plaqueta a la superficie, cambio de forma y agregación plaquetaria. En el acero AISI 316L se registra una gran cantidad plaquetas adheridas con cambio de forma y además de ello agregación. 


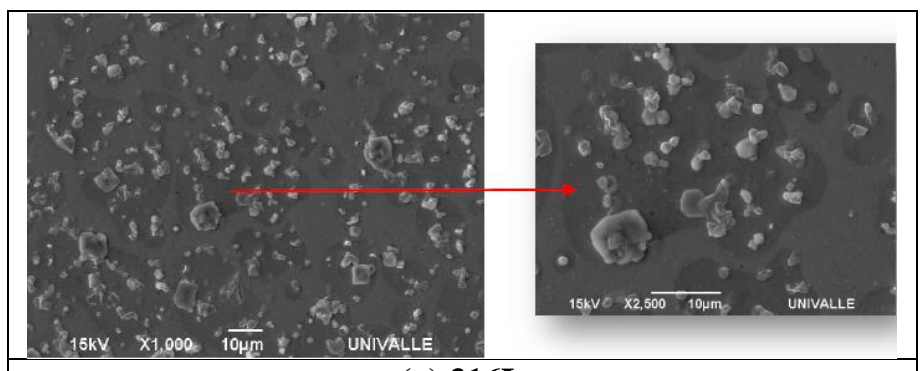

(a) $316 \mathrm{~L}$

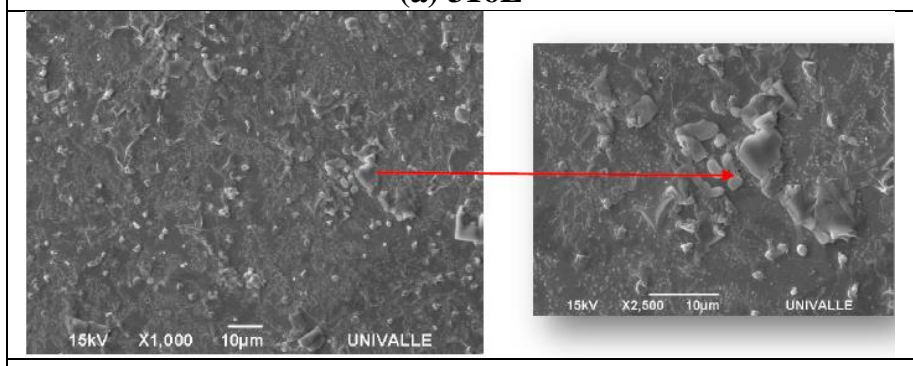

(b) $\mathbf{T i}$

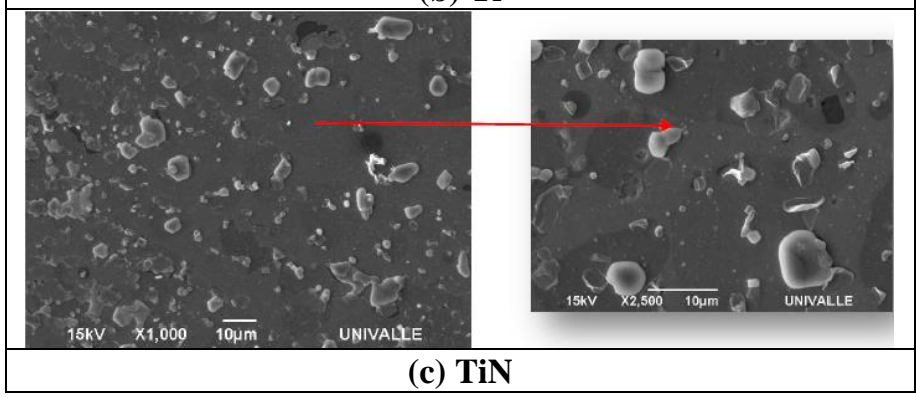

Figura 8. Micrografías de plaquetas sobre las superficies de (a) acero 316L, (b) Ti y (c) TiN.

En el titanio no solo se distingue las plaquetas adheridas sino que hay varias zonas de agregación que supera en un $45 \%$ respecto a los otros materiales utilizados. Si se compara la agregación y adhesión en los recubrimientos de TiN con el recubrimiento de Ti y el acero AISI 316L, este presenta un menor grado de agregación y adhesión, este debido a su característica cerámica, la cual se relaciona con su baja rugosidad. En la figura 9 se muestra la densidad de plaquetas por milímetro cuadrado para el sustrato de acero AISI $316 \mathrm{~L}$ y los recubrimientos de $\mathrm{Ti}$ y $\mathrm{TiN}$; se observa, que el recubrimiento de Ti promueve un mayor número de plaquetas adheridas sobre su superficie haciéndolo un material poco apto para mejorar las propiedades hemocompatibles del acero utilizado. Este comportamiento se puede correlacionar con la alta tensión interfacial Ti-Sangre con valor de $10.51 \mathrm{~nJ} / \mathrm{cm}^{2}$ que supera al recubrimiento de Ti y el sustrato.

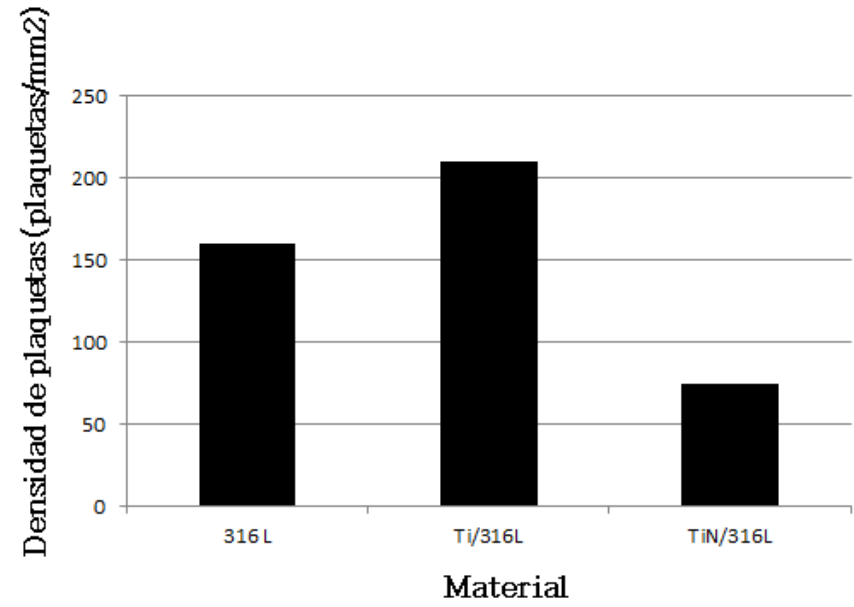

Figura 9. Densidad plaquetaria sobre las superficies materiales

\section{CONCLUSIONES}

Usando simulación computacional, se demostró la tendencia hidrofóbica de las superficies de los recubrimientos Ti hexagonal y TiN cúbico centrado en las caras, condición para la interacción del material con proteínas para aplicaciones biomédicas. Los análisis de hemocompatibilidad por agresión plaquetaria y energía superficiales, mostro una mejoría del orden del $45 \%$ para los recubrimientos de $\mathrm{TiN}$, comparado con el recubrimiento de $\mathrm{Ti}$ y el sustrato biocompatible de acero inoxidable AISI 316L. El comportamiento hemocompatible del Ti se puede correlacionar con la alta tensión interfacial que existe entre este material y la sangre.

\section{AGRADECIMIENTOS}

Los autores agradecen al Grupo de Investigación de Farmacología (In- Vitro) de la Universidad del Valle, Colombia, por su colaboración en el presente trabajo de investigación.

\section{BIBLIOGRAFIA}

[1] Young-Min Kong, Chang-Jun Bae, Su-Hee Lee, Hae-Won Kim, Hyoun-Ee Kim "Improvement in biocompatibility of $\mathrm{ZrO} 2-\mathrm{Al} 2 \mathrm{O} 3$ nano-composite by addition of HA" Biomaterials 26 (2005) 509-517

[2] Yunchang Xin, Chenglong Liu, Kaifu Huo, Guoyi Tang, Xiubo Tian, Paul K. Chu "Corrosion behavior of $\mathrm{ZrN} / \mathrm{Zr}$ coated biomedical AZ91 magnesium alloy" Surface \& Coatings Technology 203 (2009) 2554-2557

[3] D.V. Shtanskya, N.A. Gloushankova, A.N. Sheveiko, M.A. Kharitonova, T.G. Moizhess, E.A. Levashov, F. Rossi "Design, characterization and testing of Ti-based multicomponent coatings for load-bearing medical applications" Biomaterials 26 (2005) 2909-2924 
[4] M. Hamdiu, S. Hakamata, A.M. Ektessabi "Coating of hydroxyapatite thin film by simultaneous vapor deposition" Thin Solid Films 377-378 (2000) 484-489

[5] Y. F. Zheng, D. Liu, X.L. Liu, L. Li. "Enhanced corrosion resistance of $\mathrm{Zr}$ coating on biomedical TiNi alloy prepared by plasma immersion ion implantation and deposition". Applied Surface Science 255 (2008) 512-514.

[6] D. M. Devia, J. Restrepo, A. Ruden, J. González, F. Sequeda, P. J. Arango "The Tribological Characteristics of TiN, TiC, TiC/TiN Films Prepared by Reactive Pulsed Arc Evaporation Technique" Rev. Society of Vacuum Coaters (2009) 32-36.

[7] D. F. Arias, Y.C. Arango, A. Devia. "Study of TiN and $\mathrm{ZrN}$ thin films grown by cathodic arc technique". Applied Surface Science 253 (2006) 1683-1690.

[8] S. Wilson, A.T. Alpas. "Tribo-layer formation during sliding wear of TiN coatings" Wear 245 (2000) 223-229

[9] M. J. Frisch Gaussian 3, G. W. Trucks, H. B. Schlegel, G. E. Scuseria, M. A. Robb, J. R. Cheeseman, V. G. Zakrzewski, J. A. Montgomery, Jr., R. E. Stratmann, J. C. Burant, S. Dapprich, J. M. Millam, A. D. Daniels, K. N. Kudin, M. C. Strain, O. Farkas, J. Tomasi, V. Barone, M. Cossi, R. Cammi, B. Mennucci, C. Pomelli, C. Adamo, S. Clifford, J. Ochterski, G. A. Petersson, P. Y. Ayala, Q. Cui, K. Morokuma, N. Rega, P. Salvador, J. J. Dannenberg, D. K. Malick, A. D. Rabuck, K. Raghavachari, J. B. Foresman, J. Cioslowski, J. V. Ortiz, A. G. Baboul, B. B. Stefanov, G. Liu, A. Liashenko, P. Piskorz, I. Komaromi, R. Gomperts, R. L. Martin, D. J. Fox, T. Keith, M. A. Al-Laham, C. Y. Peng, A. Nanayakkara, M. Challacombe, P. M. W. Gill, B. Johnson, W. Chen, M. W. Wong, J. L. Andres, C. Gonzalez, M. Head-Gordon, E. S. Replogle, and J. A. Pople, Gaussian, Inc., Pittsburgh PA, 2001.

[10] J. K. Nørskov, T. Bligaard, J. Rossmeisl y C. H. Christensen. "Towards the computational design of solid catalysts". Nature Chem. (2000)1- 37.

[11] C. Kittel. "Introducción a la física del estado sólido" 3er Ed. Editorial Reverté S.A., (2001).

[12] A. Ruden, J. M. González, E. Restrepo. “Computational simulation of the CrN - FCC structure" Informador Técnico (Colombia) Volumen 77, No. 1 (2013) 11 - 16

[13] Software Meazure 2.0.158, página de descarga: http://meazure.softonic.com/, Abril 11 del 2011

[14] A. Ruden, Juan Manuel González Carmona, Diana Marcela Devia Narváez. "Simulación computacional de celdas cristalinas de los sistemas en películas delgadas $\mathrm{Cr}, \mathrm{c}-\mathrm{BN}$, h-
BN y TiN/TiC" Rev. El Hombre y la Máquina No. 34 (2010) 100-105.

[15] Alexander Ruden, PATENTE. "Recubrimiento Biocompatible tipo multicapa - película delgada como tratamiento superficial de sustratos biomédicos y procesos de fabricación del mismo" Universidad del Valle, Valle del Cauca. Colombia. Código: 13-138093. (2013).

[16] C.E. Wen, Y. Yamada, P.D. Hodgson. "Fabrication of novel TiZr alloy foams for biomedical applications" Materials Science and Engineering C 26 (2006) 1439 - 1444.

[17] Devia A, V. Benavides, E. Restrepo, D.F. Arias, R. Ospina. "Influence substrate temperature on structural properties of TiN/TiC bilayers produced by pulsed arc techniques". Vacuum 81 (2006) 378-384.

[18] N. Huang,P. Yang, Y.X. Leng, J.Y. Chen, H. Sun, J. Wang, G.J. Wang, P.D. Ding, T.F. Xi, Y. Leng "Hemocompatibility of Titanium Oxide Films" Biomateriales 24 (2003) 2177-2187.

[19] D. Owens; R. Wendt, "Estimation of the Surface Free Energy of Polymers”. In: J. Appl. Polym. Sci 13 (1969), P. 1741-1747.

[20] Chen JY, Leng YX, tian XB, Wang LP, Huang N, Chu PK "Antithrombogenic investigation of surface energy and optical bandgap and hemocompatibility mechanism of Ti(Ta 5)O2 thin films" Biomaterials 23 (2002) 2545-2552.

[21] A.A.A. de Queiroz, 1.R. Barrak, S.C. de Castrob "Thermodynamic analysis of the surface of biomaterials" Journal of Molecular Structure (Theochem) 394 (1997) 27 I279.

[22] Anna Waterhouse, Steven G. Wise, Martin K.C. Ng, Anthony S. Weiss. "Elastin as a Nonthrombogenic Biomaterial" Tissue Engineering Part B: Reviews. April 2011, 17(2): 93-99. 